\title{
Okadaic Acid Suppresses Melanogenesis via Proteasomal Degradation of Tyrosinase
}

\author{
Hyo-Soon Jeong, ${ }^{a}$ Hye-Young Yun, ${ }^{a}$ Kwang Jin Baek, ${ }^{a}$ Nyoun Soo Kwon, ${ }^{a}$ \\ Kyoung-Chan Park, ${ }^{b, c}$ and Dong-Seok Kim* ${ }^{*, a}$ \\ ${ }^{a}$ Department of Biochemistry, Chung-Ang University College of Medicine; 221 Heukseok-dong, Dongjak-gu, Seoul \\ 156-756, Republic of Korea: ${ }^{b}$ Department of Dermatology, Seoul National University College of Medicine; 28 \\ Yongon-dong, Chongno-gu, Seoul 110-744, Republic of Korea: and ${ }^{c}$ Department of Dermatology, Seoul National \\ University Bundang Hospital; 166 Gumi-ro, Bundang-gu, Seongnam-si, Gyeonggi-do 463-707, Republic of Korea. \\ Received March 1, 2013; accepted June 21, 2013
}

\begin{abstract}
Okadaic acid is a $\mathrm{C}_{38}$ fatty acid derivative that is known to specifically inhibit the activity of protein phosphatase 2A (PP2A). Previously, we reported that inhibition of PP2A by okadaic acid elicited extracellular signal-regulated kinase (ERK) activation, and that PP2A may be involved in melanogenesis. However, the effects of okadaic acid on melanogenesis have not been completely evaluated. In the present study, we investigated the molecular mechanisms involved in okadaic acid modulation of melanin synthesis in a spontaneously immortalized mouse melanocyte cell line, Mel-Ab. Treatment with okadaic acid inhibited melanin production in a dose-dependent manner. Moreover, okadaic acid led to a decrease in tyrosinase protein levels without altering mRNA expression. Therefore, we investigated whether the decreased level of tyrosinase by okadaic acid was related to proteasomal degradation of tyrosinase. We found that MG132, a proteasome inhibitor, almost completely abolished both the downregulation of tyrosinase levels and the inhibition of melanin synthesis by okadaic acid. Taken together, our data indicate that okadaic acid inhibits melanin synthesis via proteasomal degradation of tyrosinase.
\end{abstract}

Key words melanocyte; melanogenesis; tyrosinase; okadaic acid

The color of human skin is mainly due to the pigment melanin, which is produced within melanosomes, a specialized intracellular organelle of melanocytes. Melanogenesis is promoted by various stimulators such as UV irradiation, cytokines, growth factors, and hormones. ${ }^{1-3)}$ Tyrosinase is the critical enzyme involved in melanogenesis as it catalyses the hydroxylation of tyrosine to 3,4-dihydroxyphenylalanine (DOPA) and the oxidation of DOPA to dopaquinone. ${ }^{1,3)}$ Thus, melanin synthesis is regulated predominately by the expression and activity of tyrosinase.

Microphthalmia-associated transcription factor (MITF) is known to regulate the expression of genes related to diverse biological processes such as proliferation, survival, and pigmentation. ${ }^{4)}$ MITF is a key transcription regulator that binds to the $\mathrm{M}$ box of the tyrosinase promoter and controls the expression of tyrosinase. ${ }^{5,6}$ Moreover, MITF expression has been reported to be involved in the Wnt pathway. Activation of the Wnt signaling pathway induces glycogen synthase kinase $3 \beta$ (GSK $3 \beta$ ) inactivation (phosphorylation) and sequential $\beta$-catenin accumulation. ${ }^{7)}$ Accumulated $\beta$-catenin then binds the lymphoid-enhancing factor/T-cell factor (LEF/TCF) in the nucleus, thereby ultimately enhancing MITF expression. ${ }^{8,9)}$ In contrast, GSK $3 \beta$ is known to phosphorylate MITF at Ser298 and augment the affinity of MITF for the tyrosinase promoter, resulting in increased tyrosinase mRNA expression. ${ }^{10)}$

Okadaic acid is a $\mathrm{C}_{38}$ fatty acid $\left(\mathrm{C}_{44} \mathrm{H}_{68} \mathrm{O}_{13}\right)$ derivative that specifically inhibits the activity of protein phosphatase $2 \mathrm{~A}$ (PP2A), a serine/threonine phosphatase. PP2A has been reported to regulate the activity of extracellular signal-regulated kinase (ERK) family kinases. ${ }^{11-13)}$ We previously reported that inhibition of PP2A by okadaic acid elicits ERK activation and that there may be an association between melanogenesis and

The authors declare no conflict of interest
PP2A regulation. ${ }^{14,15)}$ However, the effects of okadaic acid on melanogenesis have not been completely investigated. In the present study, we examined the molecular mechanisms involved in okadaic acid regulation of melanin synthesis in Mel-Ab cells.

\section{MATERIALS AND METHODS}

Reagents and Antibodies Okadaic acid, 12- $O$-tetradecanoylphorbol-13-acetate (TPA), cholera toxin (CT), Triton X100, Tris, $\beta$-mercaptoethanol, phenylmethylsulfonyl fluoride, synthetic melanin, $\alpha$-melanocyte-stimulating hormone $(\alpha$ $\mathrm{MSH}$ ), and L-DOPA were all purchased from Sigma (St. Louis, MO, U.S.A.). MG132 was obtained from Calbiochem (San Diego, CA, U.S.A.). Okadaic acid and MG132 were dissolved in dimethyl sulfoxide (DMSO), and $\alpha$-MSH in distilled water. Fetal bovine serum (FBS) was purchased from Hyclone (Logan, UT, U.S.A.) and the protease inhibitor cocktail Complete $^{\mathrm{TM}}$ was obtained from Roche (Mannheim, Germany). Antibodies used in this study included phospho-specific ERK1/2 (Thr202/Tyr204, no. 9101), phospho-specific Akt (Ser473, no. 9271), phospho-specific GSK3 $\beta$ (no. 9336), total GSK $3 \beta$ (no. 9315), and $\beta$-catenin (no. 9581), which were from Cell Signaling Technology (Beverly, MA, U.S.A.). Microphthalmia Ab-1 (C5, MS-771-P0) was purchased from NeoMarkers (Fremont, CA, U.S.A.) while tyrosinase (C-19) and actin (I-19) antibodies were from Santa Cruz Biotechnology, Inc. (Santa Cruz, CA, U.S.A.).

Cell Culture For many experiments in this study we used Mel-Ab cells, a spontaneously immortalized mouse melanocyte cell line that synthesizes large quantities of melanin. ${ }^{16)}$ Mel-Ab cells were cultured in Dulbecco's modified Eagle's medium (DMEM) supplemented with 10\% FBS, $1 \mathrm{~nm}$ CT, $100 \mathrm{~nm}$ TPA, 100 units $/ \mathrm{mL}$ penicillin, and $100 \mu \mathrm{g} / \mathrm{mL}$ 
streptomycin at $37^{\circ} \mathrm{C}$ in $5 \% \mathrm{CO}_{2} . \mathrm{B} 16 \mathrm{~F} 10$ murine melanoma cells were cultured in DMEM supplemented with $10 \%$ FBS, $100 \mathrm{units} / \mathrm{mL}$ penicillin, and $100 \mu \mathrm{g} / \mathrm{mL}$ streptomycin at $37^{\circ} \mathrm{C}$ in $5 \% \mathrm{CO}_{2}$.

Cell Viability Assay Cell viability was estimated using a crystal violet assay. ${ }^{17)}$ After treating Mel-Ab or B16F10 cells with okadaic acid for $4 \mathrm{~d}$ or $3 \mathrm{~d}$, respectively, cells were stained with $0.1 \%$ crystal violet in $10 \%$ ethanol for $5 \mathrm{~min}$ at room temperature and then rinsed four times with distilled water. The crystal violet retained by adherent cells was extracted with $95 \%$ ethanol and cell viability was analyzed by measuring absorbance at $590 \mathrm{~nm}$ with an enzyme-linked immunosorbent assay (ELISA) reader (VERSAMax; Molecular Devices, Sunnyvale, CA, U.S.A.).

Measurement of Melanin Content Mel-Ab cells were incubated with okadaic acid for 3 or $4 \mathrm{~d}$, observed under a phase-contrast microscope (Olympus Optical Co., Tokyo, Japan), and photographed using a DCM300 digital camera (Scopetek, Inc., Hangzhou, China) supported by ScopePhoto software (Scopetek, Inc.). Melanin content was analyzed as described previously. ${ }^{17)}$ Briefly, cells were treated with okadaic acid and then harvested. The resulting cell pellets were dissolved in $1 \mathrm{~mL}$ of $1 \mathrm{~N} \mathrm{NaOH}$ at $100^{\circ} \mathrm{C}$ for $30 \mathrm{~min}$ and centrifuged for $20 \mathrm{~min}$ at $16000 \times \mathbf{g}$. The optical densities (ODs) of the supernatants were assessed at $400 \mathrm{~nm}$ using an ELISA reader. Standard curves of synthetic melanin $(0-300 \mu \mathrm{g} / \mathrm{mL})$ were prepared in triplicate for each experiment. B16F10 cells were incubated in six-well plates at a density of $5 \times 10^{4}$ cells/ well for $24 \mathrm{~h}$. The cells were treated with okadaic acid and incubated for $3 \mathrm{~d}$ in the presence of $\alpha$-MSH $(1 \mu \mathrm{M})$. The supernatant was analyzed at $400 \mathrm{~nm}$ and the number of cells was then enumerated using a hemocytometer.

Western Blot Analysis Cells were lysed in cell lysis buffer containing $62.5 \mathrm{~mm}$ Tris- $\mathrm{HCl} \mathrm{pH} 6.8,2 \%$ sodium dodecyl sulfate (SDS), $5 \% \beta$-mercaptoethanol, $2 \mathrm{~mm}$ phenylmethylsulfonyl fluoride, protease inhibitor cocktail, $1 \mathrm{~mm}$ $\mathrm{Na}_{3} \mathrm{VO}_{4}, 50 \mathrm{~mm} \mathrm{NaF}$, and $10 \mathrm{~mm}$ ethylenediaminetetraacetic acid (EDTA). Aliquots of cell lysates were separated by SDS-polyacrylamide gel electrophoresis and transferred to polyvinylidene fluoride (PVDF) membranes, which were then blocked with $5 \%$ skim milk in Tris-buffered saline containing $0.5 \%$ Tween 20 . Blots were incubated with the appropriate primary antibodies at a dilution of $1: 1000$, and then further incubated with horseradish peroxidase-conjugated secondary antibody. All blots were developed using an enhanced chemiluminescence detection system (Thermo Fisher Scientific Inc., Rockford, IL, U.S.A.).

Reverse Transcription-Polymerase Chain Reaction (RTPCR) To characterize mRNA expression, total RNA was isolated from cells using an RNeasy Mini kit (Qiagen, Valencia, CA, U.S.A.). Then, $1 \mu \mathrm{g}$ of RNA was reverse transcribed using the ImProm II Reverse Transcription System (Promega, Madison, WI, U.S.A.). The obtained cDNA was amplified with specific primers for tyrosinase (forward, 5'-GGCCAG CTT TCA GGC AGA GGT-3' and reverse, 5'-TGG TGC TTC ATGGGCAAAATC-3'). The PCR conditions for tyrosinase were 30 cycles of the following: $1 \mathrm{~min}$ at $95^{\circ} \mathrm{C}, 1 \mathrm{~min}$ at $59^{\circ} \mathrm{C}$, and $1 \mathrm{~min}$ at $72^{\circ} \mathrm{C}$. The resulting PCR products were visualized by electrophoretic separation on $1.5 \%$ agarose gels with Safe-Pinky DNA gel stain (Gendepot, Barker, TX, U.S.A.). Specific primers for glyceraldehyde-3-phosphate dehydroge- nase (GAPDH) were used as a control.

Statistical Analysis Statistical significance between groups was determined by Student's $t$-test and a significance level of $p<0.05$ was used.

\section{RESULTS}

Effect of Okadaic Acid on Cell Viability The effect of okadaic acid on cell viability was estimated using a crystal violet assay. Mel-Ab and B16F10 cells were incubated with okadaic acid for $4 \mathrm{~d}$ and $3 \mathrm{~d}$, respectively, at concentrations of 0-200 nm. Treatment with okadaic acid showed no effects on the viability of Mel-Ab cells or B16F10 cells over a concentration range of $0-100 \mathrm{~nm}$, indicating that okadaic acid is not cytotoxic to the cells at concentrations of $0-100 \mathrm{~nm}$ (Figs. 1A, B). However, okadaic acid at $200 \mu \mathrm{M}$ was found to be cytotoxic both in Mel-Ab cells and B16F10 cells.

Effects of Okadaic Acid on Melanin Synthesis To investigate the effect of okadaic acid on melanin production, Mel$\mathrm{Ab}$ cells were incubated with okadaic acid at concentrations of $0-100 \mathrm{~nm}$. Okadaic acid treatment significantly decreased the melanin content of Mel-Ab cells (Fig. 1C), indicating that okadaic acid induces obvious hypopigmentation in these cells. To confirm the effects of okadaic acid on melanogenesis, we performed the same experiments with the B16F10 cell line. B16F10 cells were treated with okadaic acid $(0-100 \mathrm{~nm})$ for $3 \mathrm{~d}$ in the presence of $\alpha$-MSH $(1 \mu \mathrm{M})$. Under these conditions, okadaic acid exhibited obvious inhibitory effects on melanin synthesis in B16F10 cells (Fig. 1D). Furthermore, Mel-Ab cells were observed under a phase contrast microscope and, as shown in Fig. 1E, were found to have reduced melanin pigmentation following okadaic acid treatment, in a dosedependent manner.

Effects of Okadaic Acid on the Signaling Pathways Involved in Melanogenesis It has been suggested that the ERK and Akt signaling pathways are involved in the regulation of melanin synthesis. ${ }^{18-20)}$ Moreover, okadaic acid has been reported to induce ERK phosphorylation. ${ }^{14)}$ Thus, we investigated whether okadaic acid treatment affected other signaling pathways, in addition to ERK, in melanocytes. We found that while okadaic acid induced ERK activation shortly after being introduced to cells, Akt activation was not altered (Fig. 2A). In addition, okadaic acid led to GSK $3 \beta$ phosphorylation, though levels of $\beta$-catenin did not change in okadaic acid-treated cells (Fig. 2A).

We next examined changes in MITF and tyrosinase protein levels following okadaic acid treatment in long-term cultures (Fig. 2B). Despite having hypopigmentary effects, okadaic acid treatment did not change MITF protein levels. In contrast, okadaic acid decreased tyrosinase protein levels after $24-48 \mathrm{~h}$ of incubation. The protein levels of tyrosinase were recovered at $72 \mathrm{~h}$ post-okadaic acid treatment. Moreover, GSK $3 \beta$ phosphorylation (inactivation) was induced in a time-dependent manner by okadaic acid (Fig. 2C). It has been reported that activated (dephosphorylated) GSK $3 \beta$ phosphorylates MITF at Ser298 and ultimately enhances tyrosinase mRNA expression in melanocytes. ${ }^{10)}$ Our results suggested that okadaic acid could reduce the mRNA level of tyrosinase and thus, we investigated the transcription level of tyrosinase mRNA by RT-PCR. However, as shown in Fig. 2D, okadaic acid did not decrease tyrosinase mRNA levels in a manner 
A

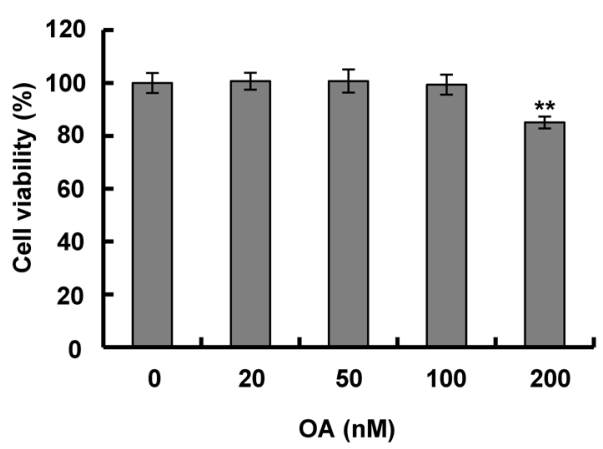

C

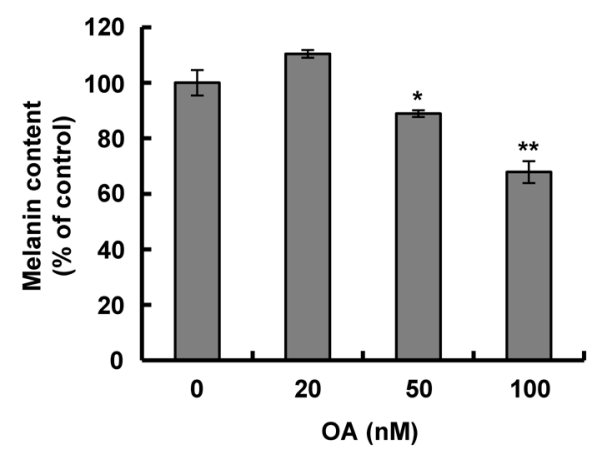

E

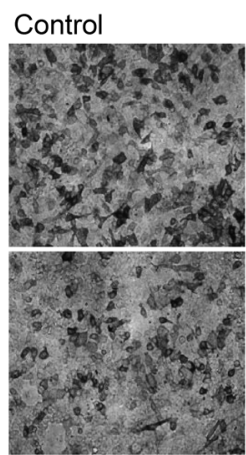

OA $50 \mathrm{nM}$
B

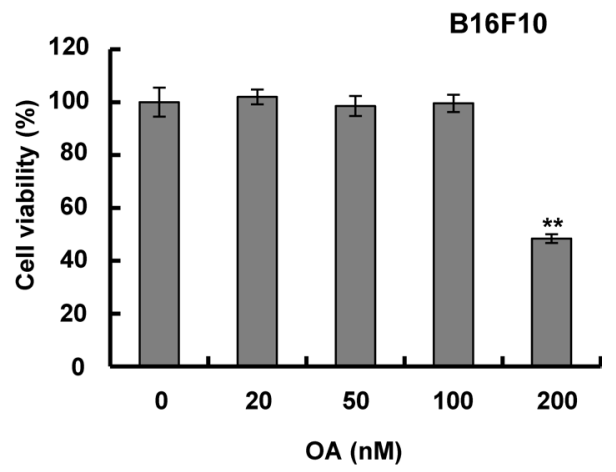

D

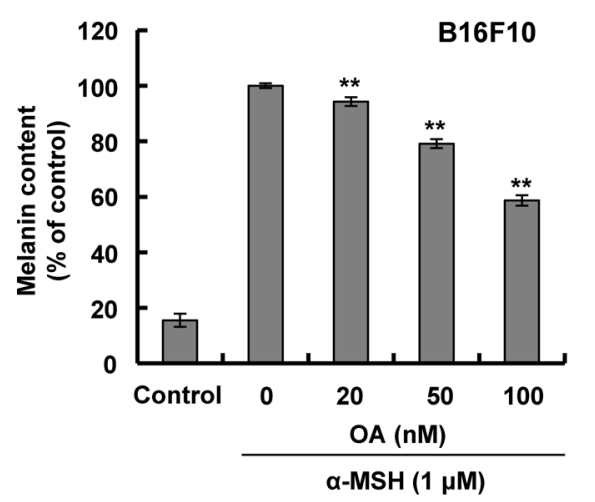

Fig. 1. Effect of Okadaic Acid on Melanogenesis

(A) Mel-Ab cells and (B) B16F10 cells were treated with okadaic acid at various concentrations (0-200nM) for $4 \mathrm{~d}$ and $3 \mathrm{~d}$, respectively. Cell viability was determined using a crystal violet assay. (C) Melanin content of Mel-Ab cells treated with okadaic acid for $4 \mathrm{~d}$ was measured as described in Materials and Methods. Data represent the mean \pm S.D. of triplicate assays expressed as percentages of the control. ${ }^{*} p<0.05,{ }^{* *} p<0.01$ compared to the untreated control. (D) B16F10 cells were treated with okadaic acid in the presence of $\alpha$-MSH $(1 \mu \mathrm{M})$ for $3 \mathrm{~d}$, and melanin content was measured as described in Materials and Methods. Data are expressed as the mean \pm S.D. of triplicate assays. ${ }^{* *} p<0.01$ compared to $\alpha$-MSH-treated B16F10 cells. (E) Mel-Ab cells were treated with $0-100$ nM okadaic acid for $4 \mathrm{~d}$, and phase contrast microscopy photographs were obtained using a digital video camera.

that was comparable to that of tyrosinase protein.

Okadaic Acid Regulates Levels of Tyrosinase via Proteasomal Degradation We next determined if the decrease in tyrosinase level was due to proteasome-mediated proteolytic degradation by okadaic acid. Melanocytes were incubated with okadaic acid for $3 \mathrm{~d}$ in the absence or presence of proteasome inhibitor MG132, after which time melanin content was determined. MG132 abrogated the inhibition of melanin synthesis by okadaic acid (Fig. 3A). In addition, MG132 completely rescued the downregulation of tyrosinase by okadaic acid (Fig. 3B). Taken together, these results indicate that okadaic acid may inhibit melanin synthesis through the proteasomal degradation of tyrosinase.

\section{DISCUSSION}

The literature indicates that there may be a relationship between PP2A activity and melanin synthesis. ${ }^{14,15)}$ Previously, we reported that heat treatment decreases pigmentation through PP2A inactivation. ${ }^{14)}$ Moreover, PP2A is involved in 
A

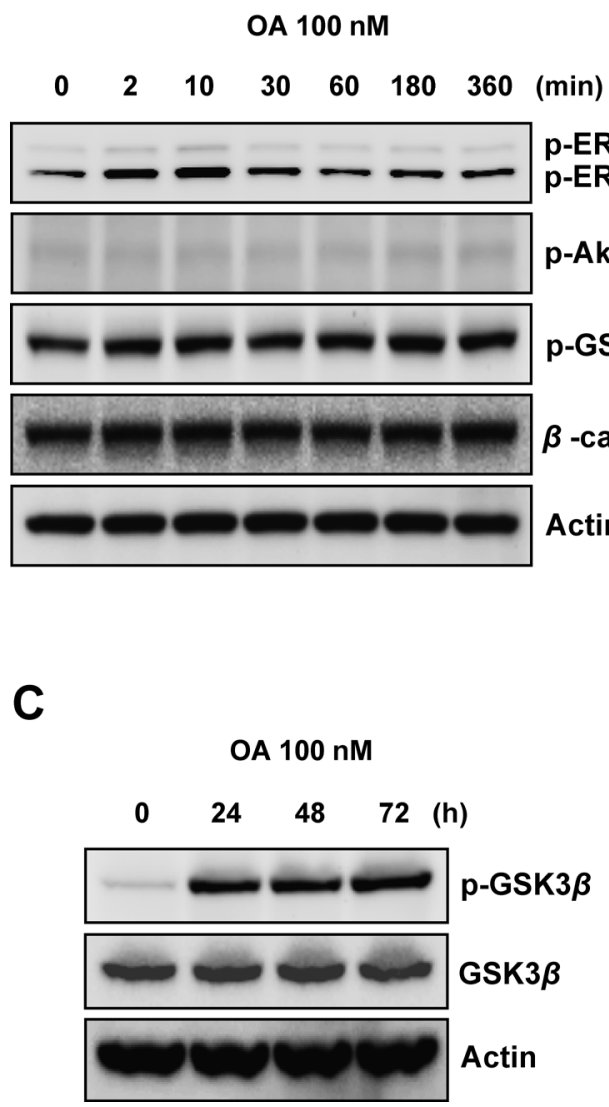

B

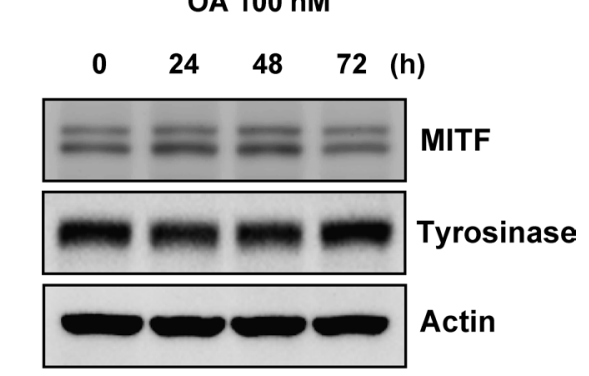

D

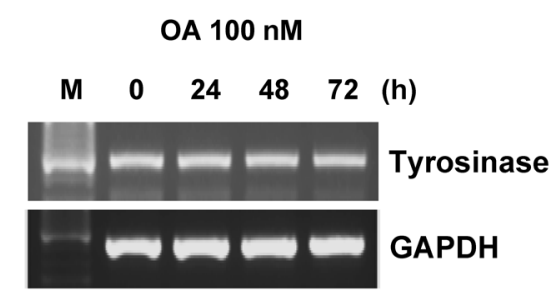

Fig. 2. Effects of Okadaic Acid on Signaling Pathways in Mel-Ab Cells

(A) After $24 \mathrm{~h}$ of serum starvation, Mel-Ab cells were treated with $100 \mathrm{~nm}$ okadaic acid for the indicated times. Whole cell lysates were then analyzed by Western blotting with antibodies against phospho-ERK, phospho-Akt, phospho-GSK3 $\beta$, and $\beta$-catenin. Equal protein loading was confirmed by use of an actin antibody. Mel-Ab cells were incubated with $100 \mathrm{~nm}$ okadaic acid for $24-72 \mathrm{~h}$. Whole cell lysates were analyzed by Western blotting with antibodies against (B) MITF and tyrosinase, or (C) phospho-GSK3 $\beta$ and total GSK3 $\beta$. Equal protein loading was confirmed by use of an actin antibody. (D) The mRNA levels of tyrosinase and GAPDH (as a loading control) were determined by RT-PCR assays as described in Materials and Methods. The resulting PCR products were analyzed by agarose gel electrophoresis. M, marker.

melanosome aggregation ${ }^{21)}$ and may also participate in melanogenesis. ${ }^{17)}$ In the present study, we investigated the effects of okadaic acid, a PP2A inhibitor, on melanin synthesis. We found that okadaic acid treatment decreased melanin content in Mel-Ab cells and B16F10 cells. These data indicate that okadaic acid has a hypopigmentary effect.

It has been previously reported that ERK activation decreased melanin synthesis via MITF downregulation. ${ }^{18,20)}$ Although okadaic acid (100 nm) slightly induced ERK activation, MITF protein levels and tyrosinase mRNA levels were not changed (Figs. 2B, D). Thus, okadaic acid-induced ERK activation may not be involved in hypopigmentation.

Active GSK $3 \beta$ is known to phosphorylate MITF at Ser298 and enhance MITF binding to the tyrosinase promoter, ultimately augmenting tyrosinase mRNA expression. ${ }^{10)}$ Okadaic acid treatment is also known to induce GSK $3 \beta$ phosphorylation (inactivation) in a model of neurodegeneration. ${ }^{22)} \mathrm{We}$ observed that okadaic acid treatment elicited GSK3 $\beta$ phosphorylation (inactivation) in Mel-Ab cells (Fig. 2C). However, we found that okadaic acid did not affect tyrosinase mRNA levels, unlike its effect on tyrosinase protein levels (Fig. 2D). Therefore, we ruled out the involvement of GSK $3 \beta$ phosphorylation in okadaic acid-induced hypopigmentation.

As okadaic acid treatment resulted in decreased tyrosinase protein levels without changing mRNA expression, it was likely that tyrosinase protein levels were being regulated by protein degradation. Thus, we investigated whether the downregulation of tyrosinase by okadaic acid was a result of proteasome-mediated proteolytic degradation. As expected, MG132, a proteasome inhibitor, almost completely abrogated the downregulation of tyrosinase levels by okadaic acid (Fig. 3B). Furthermore, MG132 treatment eliminated the decrease of melanin content by okadaic acid (Fig. 3A).

Recently, the p38 mitogen-activated protein kinase (MAPK) pathway has been reported to be involved in tyrosinase degradation. ${ }^{23)}$ Thus, we determined whether okadaic acid induces the phosphorylation of p38 MAPK in Mel-Ab cells. Although p38 MAPK was phosphorylated in okadaic acid-treated cells, SB203580, a p38 MAPK inhibitor, did not abrogate the inhibition of melanin synthesis by okadaic acid, indicating that $\mathrm{p} 38$ MAPK may not be the primary signaling pathway involved in okadaic acid-induced hypopigmentation (data not shown).

Interestingly, fatty acids have been shown to regulate melanogenesis via tyrosinase degradation. Specifically, linoleic acid (an unsaturated fatty acid) expedited the proteasomal degradation of tyrosinase, whereas palmitic acid (a saturated fatty acid) delayed this process. ${ }^{24,25)}$ Given that okadaic acid is a fatty acid derivative, our results indicate that okadaic acid may regulate the tyrosinase level via the ubiquitin-proteasome pathway. 
A

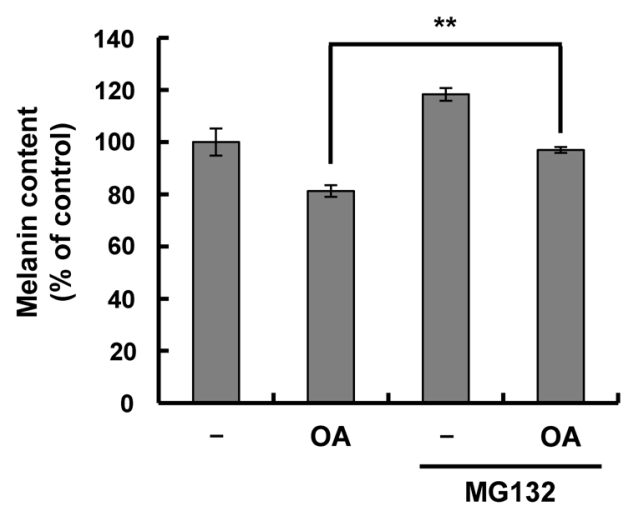

B

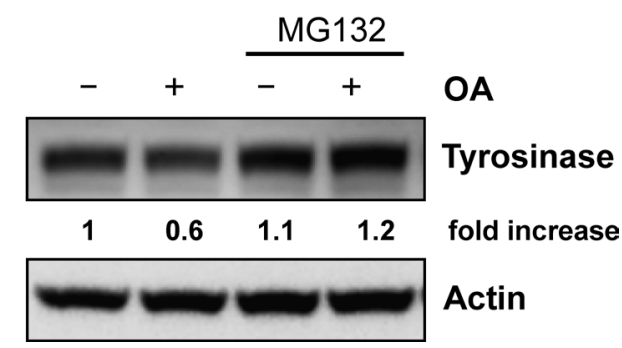

Fig. 3. Effects of MG132 on Melanin Synthesis in the Presence of Okadaic Acid

(A) Mel-Ab cells were pretreated with $80 \mathrm{~nm}$ MG132 for $2 \mathrm{~h}$ prior to the addition of $100 \mathrm{~nm}$ okadaic acid. Cells were then incubated for an additional $3 \mathrm{~d}$, after which cellular melanin content was determined. Data are expressed as the mean \pm S.D. of triplicate assays. $* * p<0.01$ compared to the okadaic acid-treated cells. (B) Mel-Ab cells were preincubated with $80 \mathrm{~nm}$ MG132 for $2 \mathrm{~h}$ prior to the addition of $100 \mathrm{nM}$ okadaic acid, and then incubated for another $48 \mathrm{~h}$. Whole cell lysates were analyzed by Western blotting with antibodies against tyrosinase and actin (loading control). Fold increases over the control levels were determined by densitometric analysis and the results are presented below each lane.

In summary, the present study evaluated the effects of okadaic acid on melanogenesis. Our results suggest that okadaic acid decreases melanin synthesis. Moreover, we found that okadaic acid downregulated the tyrosinase level via proteasomal degradation. Based on these findings, we propose that okadaic acid inhibits melanin synthesis via proteasomal degradation of tyrosinase.

Acknowledgment This study was supported by a Grant (A100179) from the Korea Healthcare Technology R\&D Project, Ministry of Health and Welfare, Republic of Korea.

\section{REFERENCES}

1) Costin GE, Hearing VJ. Human skin pigmentation: melanocytes modulate skin color in response to stress. FASEB J., 21, 976-994 (2007).

2) Schallreuter KU, Hasse S, Rokos H, Chavan B, Shalbaf M, Spencer JD, Wood JM. Cholesterol regulates melanogenesis in human epidermal melanocytes and melanoma cells. Exp. Dermatol., 18, 680-688 (2009).

3) Kondo T, Hearing VJ. Update on the regulation of mammalian melanocyte function and skin pigmentation. Expert Rev. Dermatol., 6,
97-108 (2011).

4) Harper JK, Mulgrew AE, Li JY, Barich DH, Strobel GA, Grant DM. Characterization of stereochemistry and molecular conformation using solid-state NMR tensors. J. Am. Chem. Soc., 123, 9837-9842 (2001).

5) Levy C, Khaled M, Fisher DE. MITF: master regulator of melanocyte development and melanoma oncogene. Trends Mol. Med., 12, 406-414 (2006).

6) Villareal MO, Han J, Yamada P, Shigemori H, Isoda H. Hirseins inhibit melanogenesis by regulating the gene expressions of Mitf and melanogenesis enzymes. Exp. Dermatol., 19, 450-457 (2010).

7) Larue L, Delmas V. The WNT/Beta-catenin pathway in melanoma. Front. Biosci., 11, 733-742 (2006).

8) Wu J, Saint-Jeannet JP, Klein PS. Wnt-frizzled signaling in neural crest formation. Trends Neurosci., 26, 40-45 (2003).

9) Widlund HR, Horstmann MA, Price ER, Cui J, Lessnick SL, Wu $\mathrm{M}$, He X, Fisher DE. Beta-catenin-induced melanoma growth requires the downstream target microphthalmia-associated transcription factor. J. Cell Biol., 158, 1079-1087 (2002).

10) Takeda K, Takemoto C, Kobayashi I, Watanabe A, Nobukuni Y, Fisher DE, Tachibana M. Ser298 of MITF, a mutation site in Waardenburg syndrome type 2, is a phosphorylation site with functional significance. Hum. Mol. Genet., 9, 125-132 (2000).

11) Gómez N, Cohen P. Dissection of the protein kinase cascade by which nerve growth factor activates MAP kinases. Nature, 353, 170-173 (1991).

12) Anderson NG, Maller JL, Tonks NK, Sturgill TW. Requirement for integration of signals from two distinct phosphorylation pathways for activation of MAP kinase. Nature, 343, 651-653 (1990).

13) Zhou B, Wang ZX, Zhao Y, Brautigan DL, Zhang ZY. The specificity of extracellular signal-regulated kinase 2 dephosphorylation by protein phosphatases. J. Biol. Chem., 277, 31818-31825 (2002).

14) Kim DS, Park SH, Kwon SB, Youn SW, Park ES, Park KC. Heat treatment decreases melanin synthesis via protein phosphatase $2 \mathrm{~A}$ inactivation. Cell. Signal., 17, 1023-1031 (2005).

15) Jeong HS, Park KC, Kim DS. PP2A and DUSP6 are involved in sphingosylphosphorylcholine-induced hypopigmentation. Mol. Cell. Biochem., 367, 43-49 (2012).

16) Dooley TP, Gadwood RC, Kilgore K, Thomasco LM. Development of an in vitro primary screen for skin depigmentation and antimelanoma agents. Skin Pharmacol., 7, 188-200 (1994).

17) Jeong HS, Lee SH, Yun HY, Baek KJ, Kwon NS, Park KC, Kim DS. Involvement of mTOR signaling in sphingosylphosphorylcholine-induced hypopigmentation effects. J. Biomed. Sci., 18, 1-8 (2011).

18) Kim DS, Hwang ES, Lee JE, Kim SY, Kwon SB, Park KC. Sphingosine-1-phosphate decreases melanin synthesis via sustained ERK activation and subsequent MITF degradation. J. Cell Sci., 116, 1699-1706 (2003).

19) Khaled M, Larribere L, Bille K, Aberdam E, Ortonne JP, Ballotti $\mathrm{R}$, Bertolotto C. Glycogen synthase kinase 3beta is activated by cAMP and plays an active role in the regulation of melanogenesis. J. Biol. Chem., 277, 33690-33697 (2002).

20) Kim DS, Park SH, Kwon SB, Park ES, Huh CH, Youn SW, Park KC. Sphingosylphosphorylcholine-induced ERK activation inhibits melanin synthesis in human melanocytes. Pigment Cell Res., 19, 146-153 (2006).

21) Karlsson AM, Lerner MR, Unett D, Lundström I, Svensson SP. Melatonin-induced organelle movement in melanophores is coupled to tyrosine phosphorylation of a high molecular weight protein. Cell. Signal., 12, 469-474 (2000).

22) Lim YW, Yoon SY, Choi JE, Kim SM, Lee HS, Choe H, Lee SC, Kim DH. Maintained activity of glycogen synthase kinase-3beta despite of its phosphorylation at serine-9 in okadaic acid-induced neurodegenerative model. Biochem. Biophys. Res. Commun., 395 , 207-212 (2010). 
23) Bellei B, Flori E, Izzo E, Maresca V, Picardo M. GSK3beta inhibition promotes melanogenesis in mouse B16 melanoma cells and normal human melanocytes. Cell. Signal., 20, 1750-1761 (2008).

24) Ando H, Watabe H, Valencia JC, Yasumoto K, Furumura M, Funasaka Y, Oka M, Ichihashi M, Hearing VJ. Fatty acids regulate pigmentation via proteasomal degradation of tyrosinase: a new aspect of ubiquitin-proteasome function. J. Biol. Chem., 279, 15427-15433 (2004).

25) Ando $H$, Ichihashi $M$, Hearing VJ. Role of the ubiquitin proteasome system in regulating skin pigmentation. Int. J. Mol. Sci., 10, 4428-4434 (2009). 